\author{
Military Technical College \\ Kobry El-kobbah, \\ Cairo, Egypt
}

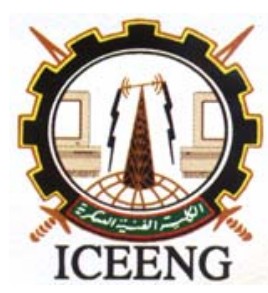

\title{
DESIGN OF AN ORBIT OF A MINI SATELLITE
}

*AA Somaie, ** Wael Badawy and **Rahim Pira

\begin{abstract}
The aim of this paper is to present the basic mathematical and theoretical knowledge of the orbit geometry system of the satellite. Information about the orbit of the satellite system is considered as the critical and main parts of the satellite design. The presented design model of the orbit geometry is valid for mini satellites (100-500 kg), which are used for imaging missions. The objective of the design model is to minimize the size, the mass, and the complexity of the satellite so that it fits the envelope of the launching vehicle. The propulsion system and others equipments of the satellite as the payload or the imaging system, on board computer system, data link and the solar cells determine the size and mass of the satellite. An example of orbit geometry for a satellite was described included the results of the design model.
\end{abstract}

Key words: Geometry, orbit control, satellite technology.

\section{Introduction}

The design procedure of a satellite is usually an iterative procedure, and can be approached from many different starting points. In this paper, we will concern about the orbit geometry of the satellite, since it can be considered as a first step to the orbit a specification of the satellite, and it has a direct impact on the physical design of the satellite in terms of the mass and volume. In simple, from the working orbit of the satellite, you can recognize its payload. In other words, if the altitude of the satellite from the earth is in between 300 to $800 \mathrm{~km}$, so it is an optical satellite, however if the altitude is over $30,000 \mathrm{~km}$ from the earth , so it is a communication satellite. The orbit of the satellite has a major influence on the design of the payload equipments. For example, in the case of low orbiting satellites, the altitude of the orbit determines the specifications of the imaging payload as the size of mirrors, focal length, etc. There are others parameters that affect the design of the satellite as the propulsion system, which is considered as a major factor in planning the size and the mass of the sat.

\footnotetext{
*Egyptian Armed Forces, aaisomaia@yahoo.com

**University of Calgary, Department of Electrical and Computer Engineering, Calgary, Canada.
} 
The fuel or the propellant of the satellite is the core of the satellite because it is used into different purposes as: (1) inject the satellite on its parking orbit; (2) correct the orbit path during flying the spacecraft; (3) disposables the satellite at the end of its lifetime [1]. The imaging system of the low orbit satellites usually works in the visible band (0.35 to 7.5 microns), where the stability and the attitude orbit control system achieves the required accuracy for imaging for the region of interest and minimizes the dead time of the imaging mission [2]. The function of the inboard computer system and the data link is to manage the imaging information and telemetry data to and from the ground station. However, the compression algorithm is considered one of the important software upon the imaging satellite since it saves memory and minimizes the time of communication when the download operation is on. The rate of the data link of the imaging satellite is one of the important parameters since it should be matched with the communication visible period between the satellite and the ground [3]. If the propellant is important to inject the satellite on its working orbit, the power supply is necessary to let the satellite live during its lifetime. The design of the solar panels, should meet the budget power that needed to all the equipment in the satellite [2].

In this paper, the orbit geometry algorithms of the satellite are described for the orbit design purpose. Since the theory and aspects including the mathematical parameters of the orbit geometry are necessary for orbit design; the following section will brief the basics of orbit geometry. Orbit geometry design model will prescribed in section III. Section IV illustrates an example of the low orbit satellite. Finally, section V contains conclusions.

\section{Aspects of orbit geometry}

In that section, we will be concerned about the necessary aspects, which have a direct impact upon the design of the orbit geometry of the imaging satellite. It is simple to start with the most important parameters and definitions of the orbit arguments. As shown in Fig. 1 (a), apogee point is the furthest point of the satellite from the earth surface or from the ground station, while perigee point is the nearest point of the satellite from the earth. The major axis is the longest diameter of an ellipse, a line that runs through the centre and both focuses, its ends being at the widest points of the shape; minor axis is the longest line that runs perpendicular to the major axis. The semi major axis is one-half of the major axis running from the center, through a focus of an ellipse, and to the edge of the ellipse as shown in Fig. 1 (b). The eccentricity $e$ is a parameter associated with every conic section [4]. It is a measure of how much a conic section deviates from being circular. For circular orbits, the eccentricity is zero while for elliptical orbits; the eccentricity is between zero and one as shown in Fig. 1 (c). In Fig. 1 (b), $a$ is the semi major axis, $b$ is the semi minor axis, and $e a$ is the linear eccentricity of the ellipse.

Some of the main angles used to describe the orientation of the orbit of the satellite are as follows:

Proceedings of the $5^{\text {th }}$ ICEENG Conference, 16-18 May, 2006

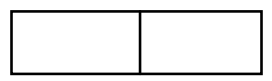


(1) Right Ascension of the Ascending Node (RAAN) angle $\Omega$ is measured in counterclockwise (eastward) from equinox Line.

(2) Argument of perigee $w$ - this is an angle, which is measured in the direction of the motion of the satellite from the ascending node to the perigee point on the line of apsides.

(3) The inclination angle $i$ represents the tilt of the orbit with respect to the equator and will have a value from 0 to 180 degrees. It is an angle measured at the ascending node, counterclockwise from Equator to orbit plane. All the above three angles are independent. Fig. 2 (a) and (b) show the right ascending angle $\Omega$, the argument of perigee $w$, and the inclination angle $i$.

(4) Eccentric Anomaly [5] - this is the angle between the direction of perigee and the current position of the satellite on its orbit, projected onto the ellipse's circumscribing circle perpendicularly to the major axis, measured at the center of the ellipse, the angle " $z c x$ ” as shown in Fig. 2 (c).

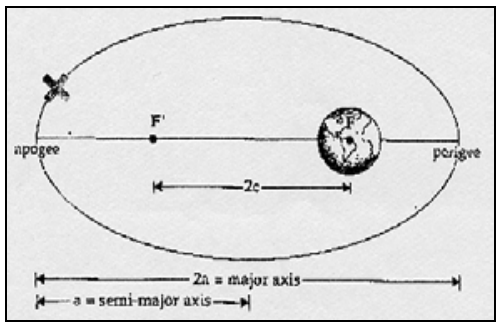

(a)

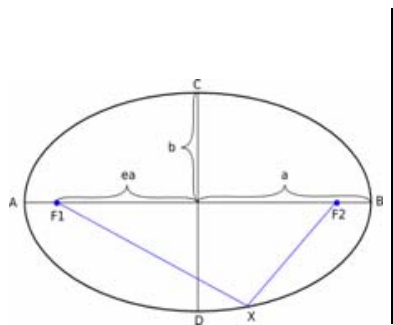

(b)

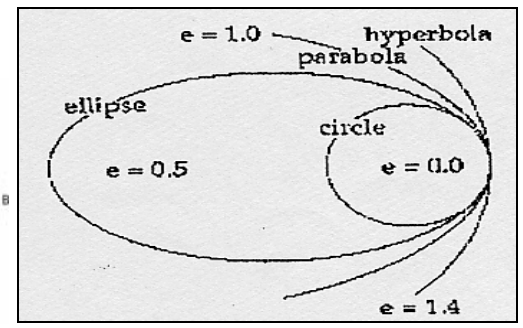

(c)

Fig.1. (a) An example of an elliptical orbit geometry, (b) the parameters of the ellipse, and (c) different orbit geometry around the earth according the value of the eccentricity " $e$ ".

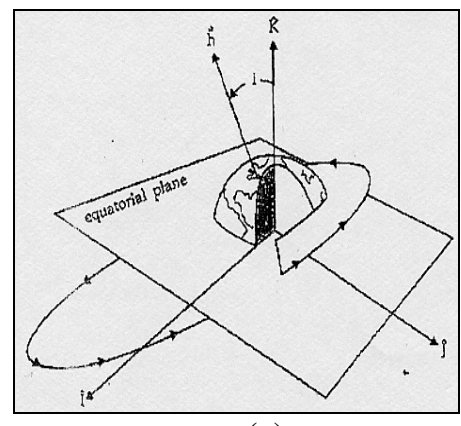

(a)

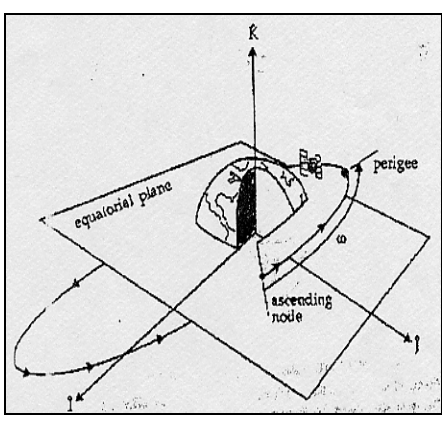

(b)

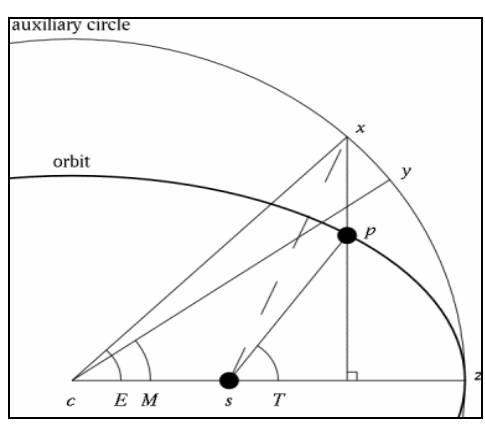

(c)

Fig. 2. An example of an elliptical orbit and the equatorial plane around the earth.

\section{Proceedings of the $5^{\text {th }}$ ICEENG Conference, 16-18 May, 2006}

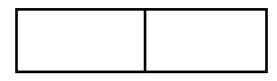




\section{Design orbit geometry model}

In this section, we will concern about the low orbit earth satellite or the imagining satellite. The first step of design an orbit for a satellite is to choose the suitable working orbit for this imaging mission. Our reference to this selection is the eclipse period and illumination conditions on the ground, investigate the effect of choosing different sun synchronous orbits, which depends upon the local solar time of the ascending node. The principal of choosing the best orbit be based on maximize the level of illumination and the shadow detail of the imaging area. This way would give some freedom for the orbit plane to drift, whilst giving reasonable viewing conditions on the ground [2]. In all cases, the initial orbital information of the launcher and the parking orbital data are important not only for design the working orbit of the satellite but also for lunching orbit.

Starting from the initial data about the orbit geometry of the satellite as the type of the orbit (circular, elliptical, or others), the altitude of the satellite, and the type of sun synchronize (synchronize or asynchronies), the Keplerian equations will be used to compute a five orbital elements or Keplerian elements. We will illustrate an example for low orbit imaging satellite in a circular orbit at altitude $h \mathrm{~km}$, and it is a sun synchronicity satellite. The sun-synchronicity is achieved by choosing exactly the right value of inclination for this orbit to ensure that the nodal precession of satellite's precisely matches the rate at which the earth orbits around the sun with its rate once per year [1]. The orbit inclination required to give the Sun-synchronicity is computed using a five orbital elements or Keplerian elements ( $a, e, i, \Omega, \omega$ ). Based on Kepler's laws, and the orbital elements shown in Fig. 3 and defined in section II, the orbit design model will be described through two algorithms orbit geometry design method, and orbit correction algorithm respectively.

\section{Orbit geometry design algorithm}

(1) The parameter $a[\mathrm{~km}]$ is semi-major axis of the elliptical orbit is calculated as,

$$
a=h+r_{m},
$$

where $h$ is the altitude of the satellite, $r_{m}$ is the mean earth radius $(\approx 6371 \mathrm{~km})$, and $a=r$ in the case of circular orbit ( $r$ and $e=0$ are the radius and eccentricity of the circular orbit respectively).

(2) The orbit period $T$ [minute] is the time at which the satellite covers or scans its orbit,

$$
\begin{aligned}
& T=2 \pi \sqrt{a^{3} / \mu}, \text { and } \\
& T=2 \pi \sqrt{a^{3} / G M},
\end{aligned}
$$

where $G$ is the gravity constant $G=6.672 \times 10^{-11} \mathrm{~m}^{3} \mathrm{~kg}^{-1} \mathrm{~s}^{-2}$, and $M$ represents the mass of the earth, $M=5.974 \times 10^{24} \mathrm{~kg}$.

\section{Proceedings of the $5^{\text {th }}$ ICEENG Conference, 16-18 May, 2006}

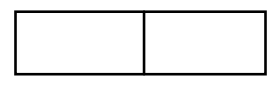

(3) The mean motion of the satellite $n$ [revolution/day] or [orbits per day], it means the number of revolution of the satellite is flying around the earth per day, 


$$
n=24 \times 60 / T=1440.0 / T \text {; }
$$

where $T$ is the orbit period in minutes.

(4) The rate of change of right-ascending node (nodal precession) equals the rate of the earth motion around the sun $d \Omega / d t=360 / 365.25=0.9856$ degree per day.

(5) Using the nodal precession rate $d \Omega / d t$ to calculate the inclination angle $i$ [degree] of the orbit as,

$$
i=\cos ^{-1}\left[\frac{-d \Omega / d t}{9.95} \times\left(a / r_{e q}\right)^{-7 / 2}\right]
$$

where $r_{e q}(\approx 6378 \mathrm{~km})$ is the equatorial radius of the earth.

(6) Since the angle $w$ represents the argument of perigee, it measures the orientation of the orbit within the orbit plane. The rate of change of argument of perigee $d w / d t$ [degree/day] is calculated as,

$$
d w / d t=-4.97\left[r_{e q} / a\right]^{3.5}\left[\left(1-5 \cos ^{2} i\right) /\left(1-e^{2}\right)^{2}\right],
$$

where the eccentricity $e=0$ in the case of the circular orbit, and the inclination angle $i$ in degree as defined in step (5).

If the rate of change of argument of perigee $d w / d t$ was negative or positive, it will lead to change the speed of the satellite to be decrease (if the $d w / d t$ positive) or increase (if the $d w / d t$ is negative), and hence loss in altitude over the lifetime and assess whether or not the departure from Sun-synchronicity is significant which make the satellite swing round dramatically. If that case was run, the propulsion system will overcome this drag effect, and the determination attitude orbit control system will correct the orbital path of the satellite. The effect of $d w / d t$ is one of the important effects of the satellite during its flying in addition to the drag effect from the residual atmosphere drifting the satellite out of its orbital path. Since this drag effect depends on the effective the cross-sectional area and the mass of the satellite, a typical drag factor $d n / d t$ might be of order $10^{-4}$ revolution per day [3]. Because of the atmospheric friction causes the satellite to lose its altitude, that drag factor causes an acceleration of the satellite to what might not be expected. It means the satellite will lose potential energy, and might be fall, so the satellite must speed up to maintain a stable orbit. In other words, as the altitude decreases, the kinetic energy of the satellite increases, however the total energy (kinetic plus potential) of the satellite is reduced. If the satellite lose its altitude, it will depart from the sun-synchronicity, thus some of the propulsion will be needed to maintain the proper altitude over the area of interest through the lifetime.

Proceedings of the $5^{\text {th }}$ ICEENG Conference, 16-18 May, 2006

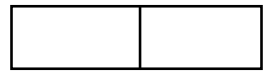


In summary, the effect of atmospheric drag is very dependent upon the altitude of the satellite as the air density falls off exponentially with height. Drag forces are strongest at perigee causing the speed of the satellite to increase at perigee rather than at apogee in the case of the elliptical orbit. Giving the lifetime of the satellite and assuming the drag factor remains at $\approx 10^{-4}$ revolution per day, then after one year, the mean motion of the satellite $n_{m}=0.0365$ revolution per day. According to the above discussion, the orbit geometry of the satellite should be corrected using the following algorithm, which is donated as orbit correction algorithm.

\section{Orbit correction algorithm}

(1) If the real motion of the satellite is $\approx 1440.0 / T$, where $T$ is orbital period, and the mean motion revolution per day is $n_{m} \approx 0.0365$, then the new motion of the satellite (after the drag factor is taken into consideration) is calculated as,

$$
n=(1440.0 / T)+n_{m}
$$

(2) Since the motion of the satellite is changed, then the corresponding orbital period in minutes $T$ will be calculated using a simple relation as,

$$
T=1440.0 / n,
$$

where the new $T$ represents period of one orbit revolution.

(3) As the orbital period $T$ of the satellite is changed, the semi-major axis $a[\mathrm{~km}]$ would be recomputed using this relation,

$$
a=\left(T^{2} \times(G M) / 4 \pi^{2}\right)^{1 / 3},
$$

Where $a$ represents the semi-major axis of the elliptical orbit or the radius of the circular orbit of the satellite, and it should be different than that one defined by equation 1 using orbit geometry design algorithm as a result of the drag effects of the rate of change apsidal rotation and the atmospheric drags.

(4) Using the inclination angle $i$, and the new $a$ (semi-major axis), the rate of change of right-ascending node $d \Omega / d t$ is computed as,

$$
d \Omega / d t=-9.95\left[r_{e q} / a\right]^{7 / 2}\left[(\cos i) /\left(1-e^{2}\right)^{2}\right]
$$

Since the eccentricity of a circular orbit equals zero, then the above equation will be modified to be as,

\section{Proceedings of the $5^{\text {th }}$ ICEENG Conference, 16-18 May, 2006}

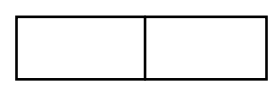




$$
d \Omega / d t=-9.95 \times[\cos i] \times\left[r_{e q} / a\right]^{7 / 2},
$$

where the unit of $d \Omega / d t$ is in degree per day, and its value should be differs than that value of the sun-synchronicity of the earth $d \Omega / d t \approx 0.9856$ degree per day (step 4 of the orbit geometry design algorithm).

(5) The drift of the rate of change of right-ascending node $d \Omega / d t$ represents the difference between the new $d \Omega / d t$ (equation 9-b), and the earth sun-synchronicity as,

$$
d=d \Omega / d t-0.9856
$$

where the unit $d$ is in degree/day.

(6) Assuming the drift of the rate of change of right-ascending node $d \Omega / d t$ is maintained constant over the lifetime of the satellite, the change in the position of the orbit plane with respect to the sun will be $\Omega^{\circ}$ as,

$$
\Omega^{\circ}=L \times d \times 365.25,
$$

where $L$ is the lifetime of the satellite, and $\Omega^{\circ}$ should be in degree.

(7) Since the sidereal day represents about 86164 seconds, and solar day equivalent to 86400 seconds, the nodal precession angle $\Omega^{\circ}$ has to change to its equivalent solar time unit using a simple relation as,

$$
\Omega=\left(86164 \times \Omega^{\circ}\right) /(360 \times 60 \times 60),
$$

Where the unit of the nodal precession angle $\Omega$ is hour and this value represents the change in the local solar time of the ascending node.

It is important to mention here, the above calculations put some limits on the accuracy of the orbit geometry. If the orbit drift represents some kilometers (difference of equation 1 and equation 8), then it causes drift from the sun-synchronicity corresponding to $\Omega$ hours (local solar time). In this case, the designer of the propulsion system of that satellite has to put into consideration like these drifts to overcome these problems, and to correct the satellite orbital orientation.

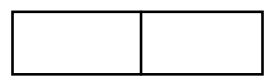




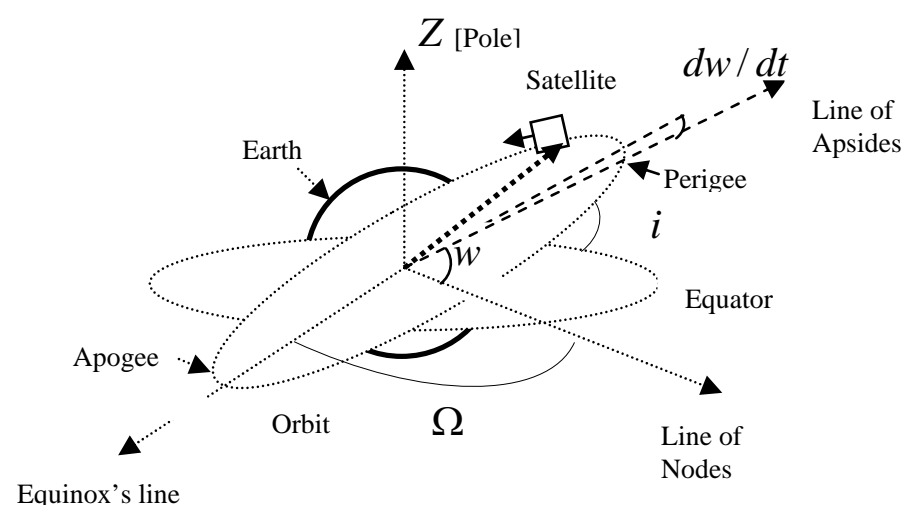

Fig. 3. The parameters of the orbit geometry of a satellite.

\section{An example and results}

In this section, we will present an example to demonstrate the orbit geometry design model. The orbit design algorithms described in the above sections were programmed using $\mathrm{C}++$, where the input data is prescribed in Table 1. The sun-synchronicity of the satellite can be achieved by choosing exactly the right value of inclination for the orbit to ensure that the nodal precession of satellite's precisely matches the rate at which the earth orbits around the sun with its rate once per year. The computation of the orbital elements of a satellite orbit geometry is based on the Keplerian elements ( $a, e, i, \Omega, \omega)$, and these elements are calculated using the orbit geometry design algorithm. It was found that the rate of change of the argument of perigee $d w / d t$ could cause some of a drag effect, if its value is positive or negative, which could be lead that the satellite loses some of its altitude. The function of orbit correction algorithm is to compute the effects of changing the orientation of the orbit within its orbit plane ( $d w / d t)$, and to correct the parameters of the orbit geometry to be match with the injection orbit or the parking orbit. It is important to add here, the orbit geometry could be needed to change, if the parking orbit is different from the injection orbit. Nerveless, similar steps will be required in addition to the method of transferring the satellite from orbit to orbit.

The preliminarily information about the defined orbit geometry (Table1) inputs to the simulated program and the results is illustrated in Table 2 and 3 for the orbit geometry design algorithm, and the orbit correction algorithm respectively.

\begin{tabular}{|l|l|}
\hline Initial orbit data & Values \\
\hline Altitude $h[\mathrm{~km}]$ & 400 \\
\hline Type of orbit & Circular \\
\hline Life time $L$ [year] & 1 \\
\hline Synchronicity & Sun- synchronize \\
\hline
\end{tabular}

Table 1. The preliminarily data of the orbit of the satellite.

Proceedings of the $5^{\text {th }}$ ICEENG Conference, 16-18 May, 2006 


\begin{tabular}{|c|l|c|}
\hline Steps & \multicolumn{1}{|c|}{ Orbit parameters } & value \\
\hline 1 & $a[\mathrm{~km}]$, semi-major axis & 6771 \\
\hline 2 & $T$ [minutes], orbital period & 92.4160 \\
\hline 3 & $n$ [revolution/day], mean motion of the satellite & 15.5817 \\
\hline 4 & $d \Omega / d t$ [degree/day], node precession & 0.9856 \\
\hline 5 & $i$ [degree], inclination angle & 97.0143 \\
\hline 6 & $d w / d t$ [degree/day], the rate of change of argument of perigee & -3.7309 \\
\hline
\end{tabular}

Table 2. The results of the orbit geometry design algorithm.

\begin{tabular}{|c|l|c|}
\hline Steps & \multicolumn{1}{|c|}{ Orbit parameters } & value \\
\hline 1 & $n$ [revolution/day], mean motion of the satellite & 15.6182 \\
\hline 2 & $T$ [minutes], orbital period & 92.1999 \\
\hline 3 & $a[\mathrm{~km}$ ], semi-major axis & 6761 \\
\hline 4 & $d \Omega / d t$ [degree/day rate of change of ascending node angle & 0.9907 \\
\hline 5 & $d$ [degree/day], difference of rate of change of ascending node angle & 0.0051 \\
\hline 6 & $\Omega^{\circ}$ [degree], nodal ascending angle & 1.8671 \\
\hline 7 & $\begin{array}{l}\Omega \text { [minutes], the corresponding change in the local solar time of the } \\
\text { ascending node }\end{array}$ & 7.4479 \\
\hline
\end{tabular}

Table 3. The results of the orbit correction algorithm.

It obvious that orbit geometry of the satellite at hand was designed and corrected using the orbit design model, since the drag effect due to the change of the rate of the argument of perigee $d w / d t$ was found to be negative value (-3.7309). The negative effect of the rate of apsidal rotation in addition the atmospheric frictions could causes a drag factor of about $\approx 10^{-4}$ revolution per day [3] which lead to increase the motion of the satellite to be about 15.6 revolution per day. Since the orbital period reduced 92.1 minutes, the semi major access decreases by $10 \mathrm{~km}$, and the satellite loses altitude, and it could depart from the sun-synchronicity. In other words, if the orbit drift is $10 \mathrm{~km}$ per year in positive or negative (high or low), then it causes drift from the sunsynchronicity corresponding to 7.4 minutes change in the local solar time of the ascending node.

\section{Conclusions}

The design of the orbit geometry of a satellite is not a simple task, since it is considered the main core of the satellite design and lunching to its working orbit. In this paper, we present a mathematical model to design and correct the orbital geometry of a satellite. It was found that a small negative drift of the argument of perigee $d w / d t$ speeding up the satellite over the planned lifetime (since it loses altitude) and suppose whether or not departure from sun-synchronicity is significant. If the orbital path of that satellite was not correctly on time using the provided propulsion system, the orbit plane could swing round dramatically.

\section{Proceedings of the $5^{\text {th }}$ ICEENG Conference, 16-18 May, 2006}

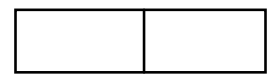


It is important to mention that the average speed of the low orbit satellite is about $7 \mathrm{~km} /$ second [4] and it fly around the earth about 15 revolutions per day. The sun-synchronicity is one of the important effects to the imaging satellite since the satellite visits the region of imaging daily at once one a time. As an example of the effect of moving the satellite from high to low orbits, the target resolution of the imaging system is inversely proportional to the altitude of the satellite.

\section{Acknowledgment}

The authors would like to thank the Laboratories for Geomatics System (LGS) and for Integrated Video System (LIVS), Departments of Geomatics Engineering, Physics and Astronomy, and Electrical and Computer Engineering at the University of Calgary for supporting this research.

\section{References}

[1] Sellers JJ, Understanding Space: An Introduction to Astronautics, McGraw Hill, (1994).

[2] Larson WJ, and Wertz JR, Space Mission Analysis and Design, $2^{\text {nd }}$ Edition, Microcosm/Kluwer, (1992).

[3] Davidoff M, “The Satellite Experimenter’s Handbook”, $2^{\text {nd }}$ Edition, ARRL, (1994).

[4] Wikipedia, the free encyclopedia, Semi major axis of an ellipse, http://en.wikipedia.org/wiki/Semi-major_axis, August, (2005).

[5] Wikipedia, the free encyclopedia, Eccentric Anomaly, http://en.wikipedia.org/wiki/Eccentric_anomaly, August, (2005). 